\title{
Capacidades metacognitivas en instituciones educativas de nivel medio
}

\section{Meta-cognitive capacity in institutions of intermediate education}

Natalie del Carmen Muñoz Morales ${ }^{1}$, Nicolás Barrientos Oradini ${ }^{2}$, Jaime Reyes Saavedra ${ }^{3}$, Albino González González ${ }^{4}$

INFORMACIÓN DEL ARTÍCULO

Fecha de recepción: 6 de Septiembre de 2018.

Fecha de aceptación: 5 de Diciembre de 2018.

Magíster en Educación. Docente Investigadora de la Universidad Miguel de Cervantes-Chile.

E-mail: natalie.muñoz@profe.umcervantes.cl Código ORCID:

https://orcid.org/0000-0002-5430-1517

${ }^{2}$ Doctor en Administración de Empresas de la Universidad Central de Nicaragua. Docente e Investigador de la Universidad Miguel de Cervantes-Chile.

E-mail: nbarrientos@umcervantes.cl Código ORCID:

https://orcid.org/0000-0002-5854-9501

3 Magíster en Educación. Docente e Investigador de la Universidad Miguel de Cervantes-Chile.

E-mail: jreyes@umcervantes.cl

Código ORCID:

https://orcid.org/0000-0002-2564-9717

4 Magíster en Educación. Docente Investigador de la Universidad Miguel de Cervantes-Chile.

E-mail: albino.gonzalez@profe.umcervantes.cl Código ORCID:

https://orcid.org/0000-0003-1574-5390

CITACIÓN: Muñoz Morales, N.C., Barrientos Oradini, N., Reyes Saavedra, J., \& González González, A. (2018). Capacidades meta-cognitivas en instituciones educativas de nivel medio. Podium, 34, 57-70 doi:10.31095/podium.2018.34.4

ENLACE DOI:

http://dx.doi.org/10.31095/podium.201 8.34 .4

\section{Resumen}

Este artículo tiene como propósito analizar las habilidades metacognitivas en estudiantes de 5to medio en instituciones de educación media en la región metropolitana de la ciudad de Santiago en Chile. La investigación se sustentó en los postulados teóricos de Caballero (2010), Woolfolk (2010), Castejón (2009), Crozier (2001), Hernán (2014), entre otros. El estudio se enmarcó como no experimental, de campo, transeccional y descriptivo. La población de estudio se conformó por un grupo de 119 estudiantes de las instituciones educativas pertenecientes al 5to grado de educación media de la comuna de Nuñoa. Los resultados mostraron que los estudiantes consideran altamente adecuadas las habilidades metacognitivas de planeación y evaluación, así como todas las diferencias individuales de la metacognición. Por lo tanto, se recomienda fortalecer la capacidad metacognitiva de los estudiantes mediante el incentivo del uso de dispositivos en el proceso enseñanza aprendizaje.

\section{Palabras Clave:}

Capacidades metacognitivas, planeación, verificación, evaluación, proceso de enseñanza aprendizaje, habilidades metacognitivas.

Clasificación JEL: M12, M14.

\begin{abstract}
This article purposes to analyze the meta-cognitive abilities of middle-fifth level students in institution of intermediate education in metropolitan area of the city of Santiago, in Chile. The research was supported, among other sources, by Caballero's (2010) theoretical postulates, Woolfolk (2010), Castejón (2009), Crozier (2001), Hernán (2014). This study was meant to be non-experimental, transactional and descriptive. The study's population was made up of a study group of 119 students of educational institutions that belong to the fifth grade of intermediate education of the Nuñoa Community. Results showed that students regard meta-cognitive abilities to be highly adequate for planning and for evaluation as well as for all individual meta-cognitive differences. It is, therefore, recommended that students' meta-cognition capacities be strengthened in order to incentivate the use of teaching and learning devices in the process.
\end{abstract}

\section{Keywords:}

Meta-cognitive capacities, planning, verification, evaluation, teaching and learning process, Meta-cognitive abilities.

JEL Classification: M12, M14.

\section{7}

PODIUM No. 34, Diciembre 2018, pp. 57-70

Universidad Espíritu Santo - UEES

ISSN: 1390-5473 e-ISSN: 2588-0969 


\section{Introducción}

El hombre se ha caracterizado por la búsqueda de formas diferentes de aprendizajes orientados a desarrollar conocimientos en su proceso evolutivo, cambiar actitudes y formas de resolver problemas, hasta llegar al punto de convertir este proceso en una condición permanente a lo largo de toda la vida. Con el transcurrir del tiempo, la tecnología se ha hecho imperativa en esa búsqueda y los seres humanos han volcado sus esfuerzos hacia su uso y apropiación en los diferentes espacios. Crozier (2001), sostiene que es conveniente modificar de manera constante las estrategias, los modelos, los ejemplos, tanto la teoría como la práctica del discurso, las formas, reglas, así como también las normas de comportamiento, pensamiento y desenvolvimiento de la vida en sus diversas manifestaciones, convirtiéndose al mismo tiempo en un reto para la adquisición de habilidades y estrategias de pensamiento que permitan alcanzar el conocimiento. En este orden de ideas, en el estudiante se debe impartir el conocimiento de tipo cognitivo, praxeológico, y actitudinal. El primero es teórico y relacionado con el saber qué y el saber por qué; el segundo está orientado a la acción, al saber cómo y a la construcción de conocimiento; y el tercero con el ser, con las actitudes propias del sujeto en relación al aprendizaje, y con el factor motivacional. Es importante debido a que se va a incrementar la brecha de calidad educativa disminuyendo la conclusión escolar y el número de estudiantes menos competentes (Necuzzi, 2013).
Es significativo entonces determinar que el enfoque metacognitivo es derivado de los estudios de las áreas de la psicología cognitiva. Se refiere al grado de conciencia o conocimiento que las personas tienen como manera de pensar que implican procesos $y$ eventos cognitivos, así como las estructuras de contenidos y la habilidad para ejercer control de dichos procesos a fin de organizarlos, revisarlos y modificarlos en función de los resultados del aprendizaje (Chadwick, 1986).

En las capacidades metacognitivas, menciona Hernán (2014), que la tecnología provee diversidad de recursos para el logro de estas, como son: Aulas virtuales, salones con video-enlaces, conexión a la red mundial y dispositivos móviles, también conocido como m-learning, que se interpreta como la concurrencia del aprendizaje electrónico con dispositivos móviles, generalmente en un marco de referencia educativo diferente al de un salón de clase y un profesor.

En relación con lo antes expuesto, en el contexto mundial Europa se ha consolidado como la región líder en cuanto a la tasa de penetración de la telefonía móvil, muy por encima de otras regiones, España tiene una tasa de penetración del 144\% según datos de la Unión Internaciones de Telecomunicaciones - UIT (2009).

De acuerdo con lo planteado por Medina y Lizardo (2013), el aprendizaje móvil busca ser la tecnología educativa del nuevo siglo, basándose en una 
instrucción formal e informal en cualquier momento o espacio geográfico dando la oportunidad de controlar el tiempo y aprovecharlo para el aprendizaje, llegando a tocar diferentes niveles y lugares en el mundo.

Es importante hacer énfasis que la creciente incorporación de los dispositivos móviles en el mundo, confirma lo que pudiese ser una necesidad social imperante por estar permanentemente comunicado con el entorno, especialmente en el caso de las nuevas generaciones o los llamados nativos digitales, ya que un estudio reciente realizado por la UNESCO (2013), señala que los jóvenes utilizan los móviles sin ninguna orientación por parte de los acudientes, y en el peor de los casos sin el adiestramiento u orientación de las instituciones educativas, es ahí donde empieza los usos indebidos de este tipo de tecnologías.

Es preciso decir que en América del Norte, gran parte de la población estudiantil emplea la tecnología de información y comunicación más como objeto de distracción y ocio que para fines educativos, los educandos no implementan tácticas de estudio apoyadas en estas herramientas, causa de ello es por el poco aprestamiento que le han suministrado las organizaciones educativas, no se les ha brindado la oportunidad de desarrollar las capacidades cognitivas con la utilización de los dispositivos móviles (Necuzzi, 2013).

\section{Paralelamente en Centroamérica,}

precisamente en México, Hernán (2014) refiere que el $84 \%$ de los estudiantes utilizan celulares o móviles en diversas actividades escolares, el 82\% para llamar, para jugar un $19 \%$, para sacar fotos un $33 \%$, el $45 \%$ escriben mensajes, un 19\% escucha música, para ver videos un $7 \%$ y el Bluetooth 13\%. Por lo tanto, al estudiante se les debe desarrollar actividades didácticas con enfoque mixto en el aprendizaje para mejorar su falta de atención, capacidad mental, maduración emotividad e interés.

En este orden de ideas, América Latina afronta relevantes desafíos en los procesos educativos, entre ellos están: Programas de capacitación del cuerpo docente insuficientes, analfabetismo en adultos, altas tasas de deserción especialmente en la educación secundaria, limitado acceso a la educación, particularmente a la educación inicial y preescolar; así lo plantea la UNESCO (2013) como causantes de la baja calidad educativa.

Es preciso destacar que estas problemáticas se manifiestan particularmente en poblaciones de bajos recursos urbanos, rurales $\mathrm{y}$ en poblaciones indígenas. Actores educativos del sector público como privado han comenzado a explorar el aprendizaje móvil para enfrentar las necesidades educativas más prioritarias de la región En América Latina y el Caribe, mientras el uso de teléfonos móviles en la sociedad está aumentando ágilmente, las iniciativas de incorporar el aprendizaje móvil se encuentran aún en niveles iniciales de desarrollo, la mayoría 
de los programas son proyectos piloto, impulsados por organizaciones sin fines de lucro o universidades, que típicamente se dirigen a pequeños grupos y focalizan en necesidades particulares o locales (UNESCO, 2012).

La calidad en los procesos de aprendizaje de la educación que la mayoría de los estudiantes obtiene en América Latina se encuentra en un nivel bajo de acuerdo con Castejon (2009), quien además indica que en las instituciones oficiales se encuentran en gran proporción los niños y niñas de básica, primaria, y secundaria, que pertenecen a estratos sociales bajos. Sus metas están encaminadas en la cobertura, disminuyendo la atención en los procesos y los resultados del aprendizaje, es decir que no se ha logrado desarrollar la transformación del sistema educativo para construir escenarios de igualación de oportunidades.

En ese sentido, Caballero (2010) afirma que los docentes logran describir de forma moderada las teorías de aprendizaje que se identifican con el uso de las tecnologías móviles en el desempeño escolar, dominan los aspectos positivos y los tipos de tecnologías, sin embargo se convierte en un desafío para poder modificar y perfeccionar, día tras día, las diferentes formas de contribuir con los procesos de enseñanza sin el respaldo de políticas educativas establecidas y estructuradas, en la búsqueda de fortalecer el quehacer pedagógico.

De allí que se pretenda analizar cómo se presentan las habilidades metacognitivas en estudiantes de 5to medio en instituciones de educación media en la región metropolitana de la ciudad de Santiago en Chile, para ampliar el conocimiento sobre la posible influencia del uso de las tecnologías de información en el desarrollo de estas capacidades.

\section{Revisión de literatura}

\section{Habilidades metacognitivas}

Es relevante abordar lo planteado por Woolfolk (2010), quien define la metacognición como la conciencia que tiene la gente sobre su propia maquinaria cognoscitiva y sobre la forma en que funciona esta. Asimismo señala que literalmente significa "conocimiento sobre los conocimientos y el aprendizaje" (pag. 54), el cual es utilizado tanto para monitorear y regular los procesos cognoscitivos como: El razonamiento, la comprensión, la solución de problemas, el aprendizaje, entre otros. A su vez implica elegir la mejor forma para manejar la tarea de aprendizaje, como lo hacen los estudiantes que tienen altas habilidades metacognitivas que organizan sus actividades, seleccionando entre varios para aprender y modifican sus estrategias si es necesario.

Por su parte Chadwick (1986) señala que las habilidades metacognitivas pueden considerarse como el nivel conocimiento que desarrollan los individuos sobre sus formas de pensar, es decir, sus procesos cognitivos, los contenidos y la habilidad orientada a 
controlar dichos procesos con la finalidad de organizarlos, revisarlos y modificarlos en función de los progresos, así como de los resultados del aprendizaje. Estas habilidades mejoran de forma importante durante las edades de niñez, tanto en lo que concierne a la memoria como en lo referente a otros ámbitos como la atención o la lectura. Necuzzi, (2013), lo explica como la consciencia que tiene el estudiante de habilidades concretas como orientación, establecimiento de un plan de acción, monitorización, dejando a un lado sus propios métodos de estudio logrando sus objetivos de aprendizaje, y un alto rendimiento en sus exámenes o controles. Del mismo modo el autor advierte que el éxito de dichas habilidades depende crucialmente de los esfuerzos del profesor para ayudar a interiorizarlas en los alumnos, y de esa forma evitar efectos que tengan relación con el desgaste emocional en los estudiantes y que conlleve a afectar el rendimiento académico (Caballero, Abello, y Palacio, 2007).

\section{Planeación}

Según Woolfolk (2010), la planeación implica cuánto tiempo dedicar a una tarea, qué estrategias utilizar, cómo empezar, qué recursos reunir, qué orden seguir, qué revisar de forma superficial y a qué ponerle mayor atención. Quiere decir entonces que planificar implica anticiparse a las acciones que han de tomarse, con el fin de lograr el aprendizaje significativo, lo cual constituyen decisiones y acciones realizadas de forma sistemática $\mathrm{y}$ ordenada, denominándose el proceso de planeación. En palabras de Chadwick (1986), es organizar los métodos o recursos a utilizar conforme a un plan y una determinada idea. La planeación exige conocer todos los sucesos, todos los recursos necesarios, todos los medios comprometidos, todos los modelos, todos los procesos, todas las posibilidades de variabilidad, todas las capacidades; sin embargo, como no es posible saber todo lo que se está haciendo, la planeación estará limitada por todo lo especificado y fundamentalmente por quien la realiza.

Por su parte Flavell (1979) explica que la planeación no sólo es una función administrativa que ayuda a señalar o programar objetivos, sino que es un criterio de vida que permite tener una perspectiva temporal de largo plazo para las actividades y metas. De la misma manera implica un conocimiento $\mathrm{o}$ contacto personal con el núcleo más íntimo del estudiante o persona que incluye la conjunción del pensamiento, las emociones, los valores, el cuerpo y las experiencias vitales que dan origen a sí mismo, es decir, al yo personal que sólo puede ser captado en un proceso intuitivo que transciende a la razón.

En relación a las definiciones antes planteadas, los diferentes autores coinciden en que se debe estar consciente de los recursos o herramientas a utilizar en el proceso de aprendizaje, sin embargo, Casares y Siliceo (2014), van un poco más allá especificando que se trata de un contacto con el interior de la persona para de esta manera poseer una visión a largo plazo de sus metas u objetivos preestablecidos. 
El criterio de Woolfolk (2010) permite entender el proceso de planeación como el tiempo, recursos, pasos, atención que conlleva las metas a alcanzar, permitiéndole al individuo conocer las actividades a ejecutar en el proceso de enseñanza-aprendizaje, lo cual le permitiría una mayor organización y por ende, un mayor rendimiento en todo lo que se proponga en su vida cotidiana o escolar. En palabras de Cabrera y Galán (2002), ello fortalecería los momentos de satisfacción académica en los estudiantes, permitiendo no sólo lograr los objetivos y metas escolares sino potenciar el rendimiento cognitivo, mejorando de esta manera las capacidades desarrolladas.

\section{Verificación}

Siguiendo la argumentación de Woolfolk (2010), la verificación es la conciencia continua de preguntarse la forma en que se están haciendo las cosas, de tal manera que verificar e indagar si tiene sentido lo ejecutado, si se ha tomado el tiempo suficiente para realizarlo, o si se ha estudiado lo necesario, es decir que es reafirmar que se está haciendo lo correcto, o reconstruir lo planeado para hacerlo de la manera correcta que le permita al estudiante concretar lo propuesto como pasar el examen con una buena calificación, realizar proyectos de investigación, asegurar su futuro de un modo correcto, entre otros. Necuzzi (2013) considera que es la etapa más importante pues permite el análisis y la comprensión de lo que está sucediendo, lo cual si se está logrando de forma correcta continuarlo, sino redireccionarlo para conseguir el fin deseado, convirtiéndose en una excelente oportunidad de autodescubrimiento en diferentes dimensiones, así como también en una retroalimentación, la cual finaliza con éxito siempre y cuando el estudiante sea consciente de cuáles son las variables que condicionan sus resultados.

Por otra parte, Castejón (2009) explica que aun cuando el paso anterior del proceso en la implementación de un plan en este caso de las habilidades cognitivas se realice de manera correcta, nada garantiza que el plan resultará de manera exitosa. Por lo tanto, se deben verificar las acciones a seguir preguntándose: ¿Se están llevando a cabo acciones necesarias en el tiempo correcto? ¿Se están haciendo con el nivel de calidad que se desea y se requiere? Entre otras preguntas, con el fin de asegurarse que esté siendo desarrollado de manera efectiva, brindado mejores resultados.

Partiendo de los conceptos basados en los autores antes citados, cada uno de ellos coincide en que la verificación consiste en corroborar las acciones que se están realizando para conocer si se están haciendo de una manera correcta, pero Necuzzi (2013) es un poco más específico señalando que es una etapa que toma relevancia al momento de obtener resultados positivos, puesto que es oportuna para enmendar los errores que se estén provocando o se puedan provocar. Sin embargo, Woolfolk (2010) la define como reafirmar pero a su vez asegurar gran parte del éxito de la implementación del plan de estudio, cuestionándose de manera constante, ya 
que posee factores críticos $\mathrm{u}$ objetivos específicos que marcan el progreso a lo largo de la trayectoria completa anticipándose a situaciones que puedan interrumpirla.

\section{Evaluación}

En palabras de la UNESCO (2012), la evaluación es definida como el monitoreo de los resultados; si los objetivos se definieron bien desde el comienzo del proceso, si se obtuvo el desempeño esperado, consecuencias inesperadas positivas o negativas, las cuales se convierten en fuente valiosa de aprendizaje, si se realizó la preparación adecuada, en otras palabras, es un proceso donde se emiten juicios basados en la información obtenida luego de emitidos los pasos o actividades. Woolfolk (2010) señala que consiste en hacer juicios acerca de los procesos y resultados, tanto del pensamiento como del aprendizaje, emitiéndose preguntas sobre las estrategias implementadas, las mejoras a lo realizado, la continuidad del proyecto, el grado de satisfacción una vez culminado el modelo, la tarea, el plan, entre otros, para evitar la intervención de los razonamientos espontáneos incorrectos en el aprendizaje. Necuzzi (2013) la define como el juicio explicitado o no de sus actividades cognitivas o de su producto mental, de esa forma el individuo tome decisiones para modificar o no sus actividades cognitivas, así como también sus productos, pero a su vez otros aspectos de la situación de aprendizaje en función al juicio metacognitivo, identificando dificultades que sesgan sus habilidades metacognitivas, pudiendo proponer acciones pedagógicas a implementar para ayudar a superarlas.

Particularmente Woolfolk (2010) señala algunas preguntas que para él son necesarias luego de concluido el proceso para la obtención de la información. Define evaluación como el juicio de los resultados que emitió el desarrollo del proceso metacognitivo, lo cual permite obtener información a partir de una serie de preguntas para de esta manera activar un proceso mental sobre las actividades cognitivas realizadas generando decisiones que modifiquen los productos negativos ocasionados permitiéndole al estudiante mejorar en futuros planes. A esto se suma Necuzzi (2013) que hace mención a lo relevante que es tomar atención ante las causas que producen la inadecuación de los estudiantes ante una determinada situación para de esta manera propiciarles solución.

\section{Diferencias individuales de la metacognición}

Según Woolfolk (2010), las diferencias de la metacognición son el resultado del desarrollo, debido a que el individuo crece volviéndose capaz de ejercitar el control ejecutivo sobre las estrategias, determinando si ha entendido las instrucciones o si estudió lo suficiente para recordar un conjunto de conceptos. No todas están relacionadas con la edad o maduración, sino que existe una gran variabilidad, incluso en estudiantes con el mismo nivel de desarrollo, y que posiblemente se originan por diferencias biológicas o por algunas variaciones en 
las experiencias de aprendizaje. Mientras que Castejón (2009) las define como las dificultades o problemas de aprendizaje, que a veces se asocian a ciertas expectativas, motivaciones, estilos de aprendizaje $y$ otros factores de personalidad, que constituyen los elementos a tener en cuenta al iniciar el proceso de enseñanza-aprendizaje, quiere decir entonces que representan una desviación de la normalidad y constituyen una necesidad educativa. Por su parte Crozier (2001) explica que las estas diferencias aluden a la diversidad más o menos notable que un alumno presenta al seguir el ritmo de aprendizaje de sus compañeros, cualquiera que sea el factor determinante en el proceso básico como memoria, metacognición, estrategias de aprendizaje, entre otros, es clave en su ventaja o retraso, puesto que detectan alteraciones en los diversos aspectos en el desarrollo de sus capacidades.

Castejón (2009) señala que las diferencias individuales tienen que ver específicamente con motivaciones, estilos de aprendizaje y otros factores de personalidad, lo cual difiere de lo planteado por Woolfolk (2010), quien señala que se trata más bien de factores biológicos o por variaciones en las experiencias de aprendizaje. Sin embargo, es Crozier (2001), quien explica con mayor detalle cuáles son esas diferencias individuales de la metacognición, permitiéndole definirla como esa particularidad notoria del estudiante que refleja durante el proceso de aprendizaje entre sus compañeros, lo cual le acarrea desventajas o beneficios ante el desarrollo de las diferentes asignaciones propuestas por el profesor.

\section{Orientación a la acción posterior al fracaso}

Para Crozier (2001) la orientación a la acción posterior al fracaso consiste en un estado de preocupación donde la persona no puede apartar de su mente los pensamientos irrelevantes para la tarea, vuelve una y otra vez sobre ellos, siendo incapaz de concentrarse en el paso siguiente. Siempre que trata de concentrarse, surgen constantemente estos pensamientos, que ocupan el espacio de la memoria de trabajo e impiden un desarrollo satisfactorio de la tarea. Por su parte Woolfolk (2010), explica que algunas diferencias se deben al desarrollo, ya que, según estudios, los niños pequeños tienen una memoria de trabajo limitada, pero que aumenta con la edad, sin embargo no resulta claro si tales diferencias son el resultado de cambios en la capacidad de la memoria o en la mejoría en el uso de las estrategias. En palabras de Gento y Vivas (2003), una de las diferencias individuales consiste en juicios metacognitivos donde el aprendiz tiene que hacer juicios sobre sí mismo y sobre sus tareas. Los interrogantes sobre el esfuerzo, las expectativas, las dificultades y los resultados necesariamente incluyen interacciones sociales, disposiciones motivacionales y consecuencias de aprendizaje. Todos estos juicios implican, de alguna manera, una autoevaluación de la metacognición donde los juicios de las personas sobre los aspectos de la situación de aprendizaje preceden a sus actuaciones. 


\section{Orientación a la acción frente a dudas}

Inicialmente Crozier (2001), indica que en un estado de dudas la capacidad de decidir algo se ve obstaculizada por la representación mental que la persona hace de los posibles resultados de la decisión, incluyendo los resultados remotos o improbables; resultando imposible prescindir de la información menos relevante y la persona se crea una representación excesivamente compleja de la situación. A pesar de esta evaluación de la información o quizá a causa de ella, no es capaz de actuar ni de comprometerse a una acción. En cambio, Woolfolk (2010), explica que la diferencia individual que afecta a la memoria a largo plazo es el conocimiento. Además, señala que cuando los estudiantes poseen mayores conocimientos procesal y declarativo de dominio específico son más capaces tanto de aprender como de recordar material en ese dominio, donde el interés es otro factor relacionado al desarrollo de conocimientos y de su recuerdo. Por su parte Gento y Vivas (2003), las denominan diferencias en las elecciones y acciones donde en el estudio de la metacognición aún queda por dilucidar cómo esta dirige la conducta donde pareciera que tanto los juicios como los pensamientos metacognitivos guían las decisiones en momentos cruciales del aprendizaje escolar.

Orientación a la acción frente a la volatilidad

Según Crozier (2001) la orientación a la acción frente a la volatilidad se parece a la preocupación porque está relacionada con dificultades para llevar las intenciones a la práctica, y para mantener la atención sobre la tarea, aunque en este caso, la atención no se dirige hacia el yo, sino hacia otras tareas. Incluso cuando la tarea se realiza satisfactoriamente, al individuo le resulta difícil concentrarse en ella a expensas de otras actividades. Castejón (2009) explica que una de las diferencias individuales entre los alumnos se relaciona a la noción de la diversidad, donde el concepto alude a cualquier diferencia notable que un alumno presenta al seguir el ritmo de aprendizaje de sus compañeros de edad, cualquiera que sea el factor determinante de la ventaja o el retraso, situándose principalmente en el conocimiento básico del que aprende, es decir que la diversidad de capacidades depende, no tanto de la capacidad cognitiva en sí misma sino de las habilidades cognitivas. Una de las diferencias individuales la denomina noción de necesidades educativas donde cada uno de los alumnos las presenta de manera particular, es decir cuando un estudiante afronta la situación de enseñanza-aprendizaje lo hace con arreglos a su estilo particular para aprender, a sus ideas, a sus centros de interés, a sus conocimientos previos, entre otros. En función de ello, los distintos alumnos avanzan en su aprendizaje con ritmos diferentes, con estrategias de aprendizaje distintas y con mayores o menores dificultades.

\section{Metodología del estudio}

Esta investigación se categorizó como no experimental, de campo (Tamayo, 2009), transeccional (Hernández, 
Fernández, y Baptista, 2010; Chávez, 2009) y descriptiva (Sabino, 2013; Bavaresco, 2013). La población de estudio se conformó por un grupo de 119 estudiantes de las instituciones educativas pertenecientes al 5 to grado de educación media de la comuna de Nuñoa en la ciudad de Santiago, sin discriminar entre géneros ni rendimiento estudiantil, y que utilizan las tecnologías de información para el desarrollo de sus actividades académicas, lo que permitió observar el nivel de presencia de las habilidades congnitivas. Se diseñó un cuestionario con 18 ítems medidos con una escala de frecuencia tipo Likert de cinco puntos (Siempre, casi siempre, a veces, casi nunca y nunca). Para validar la fiabilidad del instrumento se aplicó una prueba piloto a 15 estudiantes seleccionados, siendo excluidos posteriormente de la población final de estudio, obteniéndose un coeficiente Alfa de Cronbach de 0,93. Los datos fueron recolectados entre abril y junio del 2018.

\section{Resultados}

Para la interpretación de los resultados se diseñó un baremo interpretativo (ver Tabla 1) para facilitar la asignación de categorías.

Tabla 1

Categorización para el análisis de los resultados.

\begin{tabular}{lll}
\hline Escala de Valores & Alternativas & \multicolumn{1}{c}{ Categorías } \\
\hline $3.26 \ldots \ldots \ldots \ldots . .00$ & Siempre & Adecuada \\
$2.51 \ldots \ldots \ldots \ldots . .3 .25$ & Casi Siempre & Medianamente adecuada \\
$1.76 \ldots \ldots \ldots \ldots . .2 .50$ & Casi Nunca & Poco adecuada \\
$1.00 \ldots \ldots \ldots \ldots .1 .75$ & Nunca & Inadecuada \\
\hline
\end{tabular}

Fuente: Elaborado por autores.
La tabla 2 muestra la distribución de frecuencias de los indicadores de la dimensión Habilidades Metacognitivas, con respecto al indicador Planeación, el $84 \%$ de los estudiantes respondieron que siempre organizan todas las actividades asignadas en clases, buscan con tiempo las asignaciones que les coloca su docente, y siguen los pasos de forma ordenada para realizar las tareas, mientras que el $16 \%$ respondió casi siempre. También se observa que la media aritmética para este indicador es de 3,55 correspondiendo a la categoría altamente adecuado. Esta tendencia se corresponde con los postulados teóricos de Woolfolk (2010), quien refiere que la planeación implica cuánto tiempo dedicar a una tarea, qué estrategias utilizar, cómo empezar, qué recursos reunir, qué orden seguir, qué revisar de forma superficial y a qué ponerle mayor atención.

Tabla 2.

Habilidades Metacognitivas.

\begin{tabular}{lcccccc}
\hline $\begin{array}{c}\text { INDICADORES } \\
\begin{array}{c}\text { Alternativas de } \\
\text { respuestas }\end{array}\end{array}$ & Fa & Planeación & Verificación & Evaluación \\
\hline Siempre & 100 & $84 \%$ & 82 & $69 \%$ & Fa & $\%$ \\
Casi Siempre & 19 & $16 \%$ & 31 & $26 \%$ & 28 & $24 \%$ \\
Casi Nunca & 0 & $0 \%$ & 6 & $5 \%$ & 0 & $0 \%$ \\
Nunca & 0 & $0 \%$ & 0 & $0 \%$ & 0 & $0 \%$ \\
Total & 119 & $100 \%$ & 119 & $100 \%$ & 119 & $100 \%$ \\
Promedio & 3,55 & 3,33 & & 3,61 & \\
\hline $\begin{array}{c}\text { Interpretación } \\
\text { de la Media }\end{array}$ & $\begin{array}{c}\text { Altamente } \\
\text { adecuado }\end{array}$ & Adecuado & $\begin{array}{c}\text { Altamente } \\
\text { adecuado }\end{array}$ \\
\hline
\end{tabular}

Fuente: Elaborado por autores.

Además, se puede observar que en cuanto al indicador Verificación, el 69\% de los estudiantes respondieron que siempre verifican antes de presentar un 
examen todo el material leído, también la fuente del material que descargan de Internet y el tiempo que utilizan para realizar un proyecto en clase, $26 \%$ y $5 \%$ señalaron casi siempre y casi nunca, respectivamente. Su media aritmética de 3,33 , y por lo tanto es adecuado. Se infiere que coincide con los postulados teóricos de Woolfolk (2010), quien refiere que la verificación es la conciencia continua de preguntarse la manera en que se están haciendo las cosas de tal manera que verificar en indagar si tiene sentido lo ejecutado, si se ha tomado el tiempo suficiente para realizarlo o si se ha estudiado lo necesario. Es decir que es reafirmar que se está haciendo lo correcto, o reconstruir lo planeado para hacerlo de la manera correcta que le permita al estudiante concretar lo propuesto como pasar el examen con una buena calificación, realizar proyectos de investigación, asegurar su futuro de un modo correcto, entre otros.

Respecto al indicador Evaluación, el $76 \%$ de los estudiantes respondieron que siempre al terminar una actividad es evaluado su desempeño, los resultados negativos son tomados en cuenta para mejorar, y a partir de los resultados obtenidos son propuestos nuevos objetivos. Un 24\% respondió casi siempre. La media aritmética fue de 3,61 siendo altamente adecuado, tendencia que se corresponde con Woolfolk (2010), quien señala que la evaluación consiste en hacer juicios acerca de los procesos y resultados, tanto del pensamiento como del aprendizaje, emitiéndose preguntas sobre las estrategias implementadas, las mejoras a lo realizado, la continuidad del proyecto, el grado de satisfacción una vez culminado el modelo, la tarea, el plan, entre otros, para de esta manera evitar la intervención de los razonamientos espontáneos incorrectos en el aprendizaje.

En relación a las diferencias individuales de la metacognición en los estudiantes de 5to media (ver Tabla 3), sobre el indicador Orientación a la acción posterior al fracaso, el $71 \%$ de los encuestados respondieron que siempre al enfrentar una nueva experiencia en clases temen equivocarse, así como al presentar los resultados de una investigación realizada, les cuesta retomar el control; sin embargo, aprenden de los errores que cometen en clase; $29 \%$ respondió casi siempre. Su media aritmética fue de 3,41, ubicándolo como altamente adecuado, correspondiendo a los postulados teóricos de Crozier (2001), quien refiere que la orientación a la acción posterior al fracaso consiste en un estado de preocupación, donde la persona no puede apartar de su mente los pensamientos irrelevantes para la tarea, vuelve una y otra vez sobre ellos siendo incapaz de concentrarse en el paso siguiente. Siempre que trata de concentrarse, surgen constantemente estos pensamientos, que ocupan el espacio de la memoria de trabajo e impiden un desarrollo satisfactorio de la tarea.

En cuanto indicador Orientación a la acción frente a dudas, el $79 \%$ de los estudiantes respondieron que siempre toman decisiones en un proyecto de clases a pesar de las dudas que se les 
presentan, asumiendo el compromiso para realizarlas; $21 \%$ respondió casi siempre. Su media aritmética fue de 3,44, siendo altamente adecuado, correspondiendo con Crozier (2001), quien indica que, en un estado de dudas, la capacidad de decidir algo se ve obstaculizada por la representación mental que la persona hace de los posibles resultados de la decisión, incluyendo los resultados remotos $o$ improbables; resultando imposible prescindir de la información menos relevante y la persona se crea una representación excesivamente compleja de la situación. A pesar de esta evaluación de la información o quizá a causa de ella, no es capaz de actuar ni de comprometerse a una acción. En el mismo orden de ideas, se presenta el indicador Orientación a la acción frente a la volatilidad, el $82 \%$ de los estudiantes respondieron que siempre las posibles dificultades en la ejecución de un proyecto suelen preocuparse, al momento de realizar una tarea se preocupan más por las demás y la atención en otras actividades es mantenida mientras realizan una tarea; $18 \%$ respondió casi siempre. Su media aritmética fue de 3,55 ubicándolo como altamente adecuado y coincidiendo con los postulados de Crozier (2001), quien refiere que la orientación a la acción frente a volatilidad se parece a la preocupación porque está relacionada con dificultades para llevar las intenciones a la práctica, y para mantener la atención sobre la tarea, aunque en este caso, la atención no se dirige hacia el yo, sino hacia otras tareas. Incluso cuando la tarea se realiza satisfactoriamente, al individuo le resulta difícil concentrarse en ella a expensas de otras actividades.

Tabla 3

Diferencias individuales de la Metacognición.

\begin{tabular}{ccccccc}
\hline INDICADORES & $\begin{array}{c}\text { Orientación } \\
\text { a la acción } \\
\text { posterior al } \\
\text { fracaso }\end{array}$ & $\begin{array}{c}\text { Orientación } \\
\text { a la acción } \\
\text { frente a } \\
\text { dudas }\end{array}$ & $\begin{array}{c}\text { Orientación } \\
\text { a la acción } \\
\text { frente a } \\
\text { volatilidad }\end{array}$ \\
\hline Alternativas & Fa & $\%$ & Fa & $\%$ & Fa & $\%$ \\
\hline Siempre & 84 & $71 \%$ & 94 & $79 \%$ & 97 & $82 \%$ \\
Casi Siempre & 35 & $29 \%$ & 25 & $21 \%$ & 22 & $18 \%$ \\
Casi Nunca & 0 & $0 \%$ & 6 & $5 \%$ & 0 & $0 \%$ \\
Nunca & 0 & $0 \%$ & 0 & $0 \%$ & 0 & $0 \%$ \\
Total & 119 & $100 \%$ & 119 & $100 \%$ & 119 & $100 \%$ \\
\hline Promedio & 3,41 & 3,44 & 3,55 \\
\hline $\begin{array}{c}\text { Interpretación } \\
\text { de la Media }\end{array}$ & $\begin{array}{c}\text { Altamente } \\
\text { adecuado }\end{array}$ & $\begin{array}{c}\text { Altamente } \\
\text { adecuado }\end{array}$ & $\begin{array}{c}\text { Altamente } \\
\text { adecuado }\end{array}$ \\
\hline
\end{tabular}

Fuente: Elaborado por autores.

\section{Conclusiones}

En relación a las habilidades metacognitivas empleadas por los estudiantes de 5 to grado de la Institución Educativa Técnico Industrial Antonio Prieto Sede Santa María, se concluye que la gran mayoría de los escolares de la institución en contexto, perciben como altamente adecuadas las habilidades metacognitivas, a través de la planeación, verificación y evaluación. No obstante, un grupo significativo lo percibe como adecuado y una minoría poco significativa como medianamente adecuado. En función de las diferencias individuales de la metacognición se concluye que la gran mayoría de los escolares de las instituciones en contexto, las perciben como altamente adecuadas, a través de la orientación a la acción posterior al fracaso, orientación a la acción frente a las dudas y orientación a 
la acción frente a la volatilidad. No obstante, un grupo significativo lo percibe como adecuado.

Cabe destacar que en el contexto objeto de estudio, los estudiantes perciben de forma significativa el comportamiento y tendencia de los elementos componentes del uso de dispositivos móviles en el desarrollo de capacidades metacognitivas; sin embargo, es pertinente acotar que los resultados muestran algunas situaciones que deben someterse a consideración, por lo que se establecen algunas recomendaciones: a) Con respecto a las habilidades metacognitivas y con la finalidad de mantener la categoría de altamente adecuado, se recomienda reforzar en los estudiantes la planeación; para ello los profesores deben incentivarlos a organizar todas las actividades asignadas en clases, y asignárselas con tiempo. b) Estimular a los estudiantes a hacer la verificación de todo el material leído antes de presentar un examen, así como la fuente del material que bajan de Internet y el tiempo que utilizan para realizar un proyecto en clase. De igual manera, al terminar cada actividad, evaluar el desempeño, y proponer nuevos objetivos partiendo del resultado obtenido. c) En lo relativo a las diferencias individuales de la metacognición, y con la finalidad de mantener la categoría altamente adecuado, se recomienda reforzar en los estudiantes la orientación a la acción posterior al fracaso, estimulándolos a enfrentar las nuevas experiencias en clases sin temor a equivocarse, $\mathrm{y}$ ayudándolos a retomar el control en las presentaciones de los resultados de una investigación, a través de la técnica de respiración y concentración. d) En cuanto a la orientación a la acción frente a dudas, se recomienda orientar a los alumnos a que tomen sus propias decisiones en los proyectos de clases, independientemente de las dudas que se les presenten, a través del compromiso para su realización. e) Respecto a la orientación a la acción frente a la volatilidad, se recomienda, orientar a los estudiantes a afrontar las posibles dificultades en la ejecución de un proyecto, y a superar su preocupaciónn por la realización de sus tareas, mediante la estimulación de la autoconfianza y la concentración en las actividades que realizan, y f) Poner en práctica estrategias orientadas al uso de los dispositivos tecnológicos, con la finalidad de desarrollar las capacidades metacognitivas en los estudiantes analizados.

\section{Referencias}

Bavaresco, A. (2013). Proceso Metodológico en la Investigación. Cómo hacer un diseño de investigación (6ta ed.). Venezuela: Imprenta Internacional C.A.

Caballero, C.C., Abello, R., y Palacio, J. (2007). Relación del burnout y el rendimiento académico con la satisfacción frente a los estudios en estudiantes universitarios. Avances en Psicología Latinoamericana, 25(2), 98-111.

Caballero, E. (2010).Tecnologías móviles en el desempeño escolar en la educación media (tesis de grado). Universidad Rafael Urdaneta, Maracaibo, Venezuela.

Cabrera, P., y Galán, E. (2002). Satisfacción escolar y rendimiento académico. Revista de Psicodidáctica, 14, 87-97. 
Castejón, J.L. (2009). Unas bases psicológicas de la educación especial (5ta ed.). España: Editorial Club Universitario.

Chadwick, C.B. (1986). Estrategias cognitivas, metacognición y el uso de las microcomputadoras en la educación. Revista del Instituto de Investigaciones Educativas, 12(55), 21-35.

Chávez, N. (2009). Introducción a la Investigación Educativa. Venezuela: Gráfica González.

Crozier, W.R. (2001). Diferencias individuales en el aprendizaje: Personalidad y rendimiento escolar. España: Narcea S.A. de Ediciones.

Flavell, J.H. (1979). Metacognition and cognitive monitoring: A new area of cognitive-developmental inquiry. American Psychologist, 34(10), 906-911. doi:10.1037/0003-066X.34.10.906

Gento, S., y Vivas, M. (2003). El SEUE: un instrumento para conocer la satisfacción de los estudiantes universitarios con su educación. Acción Pedagógica, 12(2), 16- 27.

Hernán, C. (2014). Aprendizaje Móvil o M-Learning. De:http://mlearning2012.blogspot.com/p/ ventajas-y-desventajas.html

Hernández, R., Fernández, C., y Baptista, P. (2010). Metodología de la Investigación (5ta ed.). México DF, México: Editorial Mc GrawHill Interamericana S.A.

Medina, M., y Lizardo, A. (2013). Uso de dispositivos en el aprendizaje de los estudiantes de los programas nacionales de formación. En VII Jornadas Nacionales y IV Internacionales de Investigación de la URBE, Maracaibo, Venezuela.

Necuzzi, C. (2013). Estado del arte sobre el desarrollo cognitivo involucrado en los procesos de aprendizaje y enseñanza con integración de las TIC. Argentina: UNICEF.
Organización de las Naciones Unidas para la Educación, la Ciencia y la Cultura UNESCO. (2012). Informe regional de monitoreo del progreso hacia una educación de calidad para todos en América Latina y el Caribe, EPT 2012. Recuperado de http://unesdoc.unesco.org/images/0021/0 02158/215880s.pdf.

Organización de las Naciones Unidas para la Educación, la Ciencia y la Cultura UNESCO. (25 de febrero de 2013). Aprendizaje mediante dispositivos móviles: "No podemos seguir viviendo en la era predigital. Servicio de Prensa. Recuperado de:

http://www.unesco.org/new/es/media-ser vices/single-view/news/mobile_learning _we_cannot_continue_to_live_in_the_pr e_di/

Sabino, C. (2013). El Proceso de la Investigación. Argentina: Editorial Lumen.

Tamayo, M. (2009). El Proceso de la Investigación Cientifica (4ta ed.). México DF, México: Editorial Limusa.

Unión Internacional de Telecomunicaciones-UIT. (2009). Perfiles Estadísticos de la Sociedad de la Información 2009. Región de América. Recuperado de https://www.itu.int/dms_pub/itu-d/opb/in d/D-IND-RPM.AM-2009-E09-R1-PDFS.pdf.

Woolfolk, A. (2010). Psicología Educativa (11ava ed.). México: Pearson Educación. 